University of Nebraska - Lincoln DigitalCommons@University of Nebraska - Lincoln

2009

\title{
Quantum scattering time and its implications on scattering sources in graphene
}

X. Hong

K.Zou

J.Zhu

Follow this and additional works at: https://digitalcommons.unl.edu/physicshong

Part of the Atomic, Molecular and Optical Physics Commons, and the Engineering Physics Commons

This Article is brought to you for free and open access by the Research Papers in Physics and Astronomy at DigitalCommons@University of Nebraska Lincoln. It has been accepted for inclusion in Xia Hong Publications by an authorized administrator of DigitalCommons@University of Nebraska Lincoln. 


\title{
Quantum scattering time and its implications on scattering sources in graphene
}

\author{
X. Hong, K. Zou, and J. Zhu \\ Department of Physics, The Pennsylvania State University, University Park, Pennsylvania 16802, USA
}

(Received 18 November 2009; published 29 December 2009)

\begin{abstract}
We determine the quantum scattering time $\tau_{q}$ in six graphene samples with mobility of $4400<\mu$ $<17000 \mathrm{~cm}^{2} / \mathrm{V} \mathrm{s}$ over a wide range of carrier density $\left(1.2<n<6 \times 10^{12} / \mathrm{cm}^{2}\right)$. $\tau_{q}$ derived from Shubnikov-de Haas oscillation ranges $\sim 25-74 \mathrm{fs}$, corresponding to a single-particle level broadening of 4.5-13 meV. The ratio of the transport to quantum scattering time $\tau_{t} / \tau_{q}$ spans $1.5-5.1$ in these samples, which can be quantitatively understood combining scattering from short-ranged centers and charged impurities located within $2 \mathrm{~nm}$ of the graphene sheet. Our results suggest that charges residing on the $\mathrm{SiO}_{2}$ surface play a dominant role in limiting carrier mobility in current samples.
\end{abstract}

DOI: 10.1103/PhysRevB.80.241415

PACS number(s): 73.63.-b, 73.21.-b, 73.43.-f, 72.15.Lh

Understanding and eliminating extrinsic scattering sources in graphene is critical to the advancement of its fundamental study and technological applications. Despite many theoretical and experimental investigations into possible candidates, including charged impurities (CIs), adsorbates, substrate corrugations, and ripples, contradictory observations remain and a clear picture has yet to emerge. ${ }^{1-16}$

To date, most experimental studies have focused on probing the carrier mobility $\mu$, or equivalently the transport scattering time $\tau_{t}=m^{*} \mu / e .^{8-15}$ Another important parameter in two-dimensional (2D) transport, the quantum scattering time $\tau_{q}$, has not been well studied. $\tau_{q}$ characterizes the momentum relaxation of a quasiparticle and relates to its quantum level broadening $\Gamma$ through $\Gamma=\hbar / 2 \tau_{q}$.

Quantitatively, the difference between $\tau_{q}$ and $\tau_{t}$ in graphene is shown in the following equations: ${ }^{17}$

$$
\begin{gathered}
\frac{1}{\tau_{q}}=\int_{0}^{\pi} \mathrm{Q}(\theta)(1+\cos \theta) d \theta, \\
\frac{1}{\tau_{t}}=\int_{0}^{\pi} \mathrm{Q}(\theta)(1+\cos \theta)(1-\cos \theta) d \theta .
\end{gathered}
$$

Here, $\theta$ is the scattering angle and $\mathrm{Q}(\theta)$ depends on specific scattering mechanisms. ${ }^{17,18}$ While small-angle events weigh heavily toward $\tau_{q}, \tau_{t}$ is mostly affected by right-angle scatterings. Measurement of $\tau_{t} / \tau_{q}$ has proven to be a powerful diagnostic tool in revealing complex scattering scenarios in conventional 2D electron gases (2DEGs). ${ }^{18-21}$ For example, short-ranged scattering sources give rise to $\tau_{t} / \tau_{q} \sim 1$ while charged impurities far away from a 2 DEG lead to predominately small-angle events, resulting in large $\tau_{t} / \tau_{q}$. The former has been observed in silicon inversion layers and the latter characterizes modulation doped GaAs 2DEGs. ${ }^{18-20} \mathrm{De}-$ spite its demonstrated importance, the study of $\tau_{q}$ in graphene has been scant. Existing data are largely obtained from the linewidth of cyclotron resonance at low densities. ${ }^{22} \mathrm{~A}$ systematic comparison between $\tau_{t}$ and $\tau_{q}$ has not been made.

In this work, we report a comprehensive study of $\tau_{q}$ in six graphene samples over a wide range of carrier densities 1.2 $<n<6 \times 10^{12} / \mathrm{cm}^{2}$ and mobility $4400<\mu<17000 \mathrm{~cm}^{2} /$ V s. $\tau_{q}$ is obtained from Shubnikov-de Haas $(\mathrm{SdH})$ oscillations and ranges approximately 25-74 fs in these samples, corresponding to $\Gamma=4.5-13 \mathrm{meV}$. The $n$ dependence of $\tau_{t}$, $\tau_{q}$, and their ratio $\tau_{t} / \tau_{q}$ can all be explained by a selfconsistent Boltzmann transport theory ${ }^{3,17}$ using three parameters: the charged-impurity density $n_{i m p}$, the impuritygraphene distance $z$, and the resistivity from short-ranged scatterers $\rho_{\text {short }}$. Our results indicate that the mobility in current graphene-on- $\mathrm{SiO}_{2}$ samples is limited by scattering from charges residing within $2 \mathrm{~nm}$ of the graphene sheet. We speculate that charges present at the graphene $/ \mathrm{SiO}_{2}$ interface are the major sources of scattering.

Single-layer graphene sheets are mechanically exfoliated onto $290 \mathrm{~nm} \mathrm{SiO}_{2}$ /doped $\mathrm{Si}$ substrates and identified optically. Rectangular pieces are processed into Hall bar devices using standard e-beam lithography followed by metal deposition (Fig. 1 inset). The fabrication details are given in Ref. 23. Representative data from four samples (denoted as samples A-D) are presented in detail.

Transport experiments are performed in a pumped $\mathrm{He}^{4}$ cryostat with a base temperature of $1.4 \mathrm{~K}$ and equipped with a $9 \mathrm{~T}$ magnet. Standard lock-in techniques are used with an excitation current of 50-200 nA. The doped Si substrates serve as back gate electrodes, to which a voltage $\left(V_{\mathrm{g}}\right)$ is applied to tune the carrier density and hence the conductance of graphene. We extract carrier density from $\mathrm{SdH}$ oscillations and obtain a gating efficiency of $\alpha=\mathrm{d} n / \mathrm{d} V_{\mathrm{g}}=7$ $\times 10^{10} / \mathrm{cm}^{2} \mathrm{~V}$ of the backgate. All measurements are taken at $T<10 \mathrm{~K}$ to eliminate electron-phonon scattering.

Figure 1 shows the conductivity $\sigma$ vs $V_{\mathrm{g}}$ taken on samples

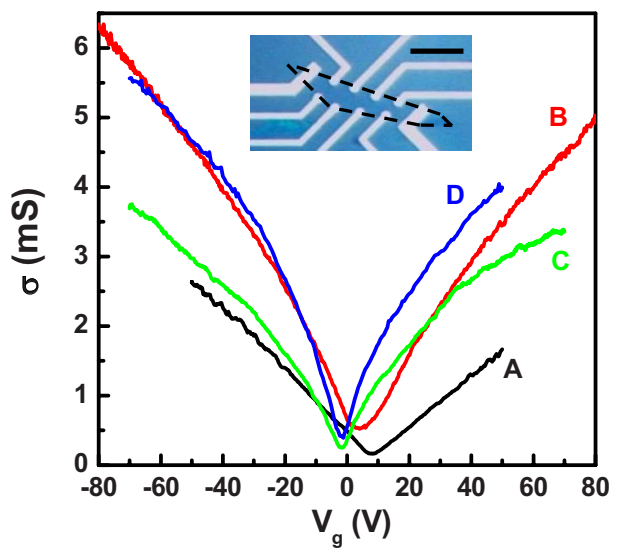

FIG. 1. (Color online) $\sigma\left(V_{\mathrm{g}}\right)$ of samples A (black), B (red), C (green), and D (blue) below $10 \mathrm{~K}$. Inset: optical image of sample A. Edge of the piece outlined. Scale bar is $10 \mu \mathrm{m}$. 
TABLE I. $\mu_{\mathrm{FE}}, \rho_{\text {short }}, \tau_{q}, \tau_{t} / \tau_{q}, z$, and $n_{\text {imp }}$ for samples A-D. $\tau_{q}$ and $\tau_{t} / \tau_{q}$ are given for $n \sim 3$ $\times 10^{12} / \mathrm{cm}^{2}$. The uncertainty in $z$ is $1-2 \AA$ for all samples. Data in parentheses are after the corrections of density inhomogeneity.

\begin{tabular}{ccccccc}
\hline \hline & $\begin{array}{c}\mu_{\mathrm{FE}} \\
\left(\mathrm{cm}^{2} / \mathrm{V} \mathrm{s}\right)\end{array}$ & $\begin{array}{c}\rho_{\text {short }} \\
(\Omega)\end{array}$ & $\begin{array}{c}\tau_{q} \\
(\mathrm{fs})\end{array}$ & $\tau_{t} / \tau_{q}$ & $\begin{array}{c}z \\
(\mathrm{~nm})\end{array}$ & $\begin{array}{c}n_{\text {imp }} \\
\left(10^{11} / \mathrm{cm}^{2}\right)\end{array}$ \\
\hline $\mathrm{A}$ & 4400 & 40 & $31(38)$ & $2.7(2.2)$ & 0 & 10.4 \\
$\mathrm{~B}$ & 10000 & 55 & $33(49)$ & $5.1(3.4)$ & $2(1)$ & $(7.7)$ \\
$\mathrm{C}$ & 9500 & 165 & 66 & 1.7 & 0 & 4.8 \\
$\mathrm{D}$ & 17000 & 105 & 53 & 3.5 & 2 & 7 \\
\hline \hline
\end{tabular}

A-D. $\sigma\left(V_{\mathrm{g}}\right)$ varies linearly with $V_{\mathrm{g}}$ for the whole density range in sample A and exhibits sublinear $\sigma\left(V_{\mathrm{g}}\right)$ at high $V_{\mathrm{g}}$ for samples B-D, where the "bowing" is most pronounced in sample C. The qualitative features of these traces resemble results reported previously ${ }^{10-13}$ and have been explained by a self-consistent Boltzmann theory combining scattering from long and short-ranged sources, ${ }^{1-3}$

$$
\frac{1}{\sigma}=\frac{1}{\sigma_{\text {long }}}+\rho_{\text {short }} ; \quad \sigma_{\text {long }}=n e \mu_{\mathrm{FE}}+\sigma_{\text {res }} .
$$

In this framework, $\rho_{\text {short }}$ denotes a constant contribution to resistivity from short-ranged scattering sources such as defects or neutral adsorbates. CI are thought to give rise to a linear $\sigma\left(V_{\mathrm{g}}\right)$, which implies a constant field effect mobility $\mu_{\mathrm{FE}}$ and consequently $\tau_{t} \propto \sqrt{n}$. Equations (2) produce excellent fittings to the $\sigma\left(V_{\mathrm{g}}\right)$ data of all our samples. The resulting $\mu_{\mathrm{FE}}$ and $\rho_{\text {short }}$ span $4400-17000 \mathrm{~cm}^{2} / \mathrm{V} \mathrm{s}$ and $40-165 \Omega$, respectively (Table I), covering much of the variations reported in the literature. The residue conductivity $\sigma_{\text {res }}$ ranges $0.1-0.35 \mathrm{mS} .^{12,24}$ In samples exhibiting electronhole asymmetry, our analyses focus on the carrier type with the higher $\mu_{\mathrm{FE}}$ to avoid complications associated with contact doping. ${ }^{25}$

Although Eqs. (2) provide a good description of existing conductivity measurements, the origin of scattering sources in graphene is still under debate. In addition to CI, ripples, resonant scatterers, and midgap states are also potential candidates. $^{4-7}$ Unlike potassium adatoms, ${ }^{12}$ certain adsorbates seem to dope graphene but not degrade its mobility. ${ }^{9}$ The role of the dielectric environment also appears controversial. ${ }^{13-15}$ While Jang et al. found agreement with the model using ice as a top dielectric layer, ${ }^{13}$ Ponomarenko et al. reported screening effects much smaller than expected from the CI model using liquid dielectric layers. ${ }^{15}$ Within the charged-impurity model, the origin of such impurities remains unclear: adsorbates on top of graphene, charges adsorbed/trapped at the graphene $/ \mathrm{SiO}_{2}$ interface or residing inside the substrate are all possible candidates.

We have measured the quantum scattering time $\tau_{q}$ in graphene to further address the above issues. We determine $\tau_{q}$ from the magnetic field dependence of $\mathrm{SdH}$ oscillations following procedures well established in conventional 2DEGs. ${ }^{20}$ Figure 2(a) shows the magnetoresistance $\rho_{\mathrm{xx}}(B)$ of sample A at $n=3.89 \times 10^{12} / \mathrm{cm}^{2}$ and $T=1.6 \mathrm{~K}$. The oscillatory amplitude $\delta \rho_{\mathrm{xx}}$ can be described by

$$
\frac{\delta \rho_{\mathrm{xx}}}{\rho_{0}}=4 \gamma_{t h} \exp \left(-\frac{\pi}{\omega_{c} \tau_{q}}\right) ; \quad \gamma_{t h}=\frac{2 \pi^{2} k_{B} T / \hbar \omega_{c}}{\sinh \left(2 \pi^{2} k_{B} T / \hbar \omega_{c}\right)} .
$$

Here $\rho_{0}$ is the nonoscillatory background resistance, $\gamma_{t h}$ is the thermal factor, and $\omega_{c}$ the cyclotron frequency in graphene. Here $m^{*}=E_{F} / v_{F}^{2}=\hbar \sqrt{\pi n} / v_{F}$ is the effective mass and $v_{F}=1 \times 10^{6} \mathrm{~m} / \mathrm{s}$ is the Fermi velocity in graphene. Figure 2(b) plots $\delta \rho_{\mathrm{xx}} / \gamma_{t h}$ vs $1 / B$ in a semilog plot (the Dingle plot), where we extract $\tau_{q}=34$ fs from the slope of the linear fit. The corresponding $\delta \rho_{\mathrm{xx}}$ calculated from Eq. (3) is plotted in Fig. 2(a) as dashed lines and exhibits excellent agreement with data. In each sample, the same procedure is repeated at different densities for $n>1.2 \times 10^{12} / \mathrm{cm}^{2}$, where several well-developed SdH oscillations are observed before the onset of quantum Hall states. In some traces, a slowly varying background is subtracted before the determination of $\delta \rho_{\mathrm{xx}}$, as described in Ref. 23.

Figure 2(c) plots $\tau_{q}(n)$ in samples A-D (also listed in Table I for $\left.n=3 \times 10^{12} / \mathrm{cm}^{2}\right) . \tau_{q}(n)$ increases with increasing $n$ in all samples and spans $25-74$ fs for $1.2 \times 10^{12} / \mathrm{cm}^{2}<n$ $<6 \times 10^{12} / \mathrm{cm}^{2}$. These values correspond to a quantum level broadening $\Gamma=4.5-13 \mathrm{meV}$ and are in line with $\Gamma$ $=20-30 \mathrm{meV}$ extracted from the adsorption linewidth of cyclotron resonances at $n<1 \times 10^{12} / \mathrm{cm}^{2}$. ${ }^{22}$

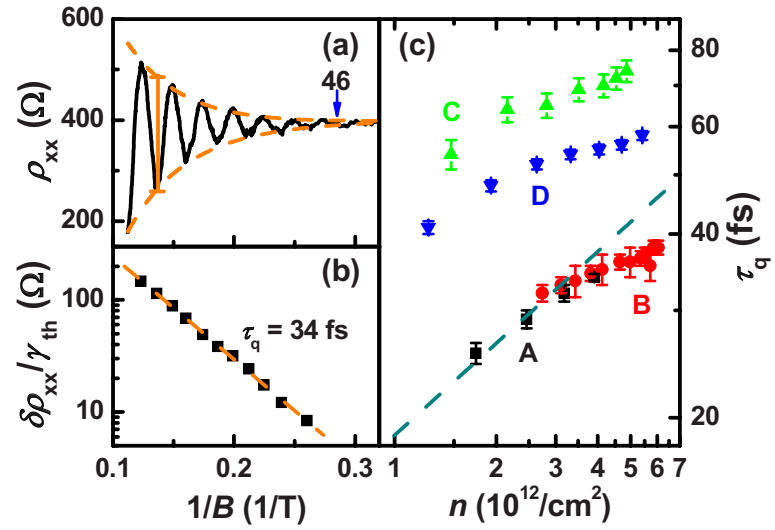

FIG. 2. (Color online) (a) $\rho_{\mathrm{xx}}(1 / B)$ of sample $\mathrm{A}$ at $1.6 \mathrm{~K}$ and $n=3.89 \times 10^{12} / \mathrm{cm}^{2}$. The onset of the oscillations corresponds to filling factor $\nu=46$ (arrow). The vertical bar marks $2 \delta \rho_{\mathrm{xx}}$ at $\nu=22$. The dashed lines correspond to Eq. (3) with $\tau_{q}=34$ fs. (b) The corresponding Dingle plot: $\delta \rho_{\mathrm{xx}} / \gamma_{t h}$ vs $1 / B . \tau_{q}$ is extracted from the linear fit. (c) $\tau_{q}(n)$ of samples A (squares), B (circles), C (up triangles), and D (down triangles) on a log-log plot. The dashed line indicates $\sqrt{n}$ dependence. 


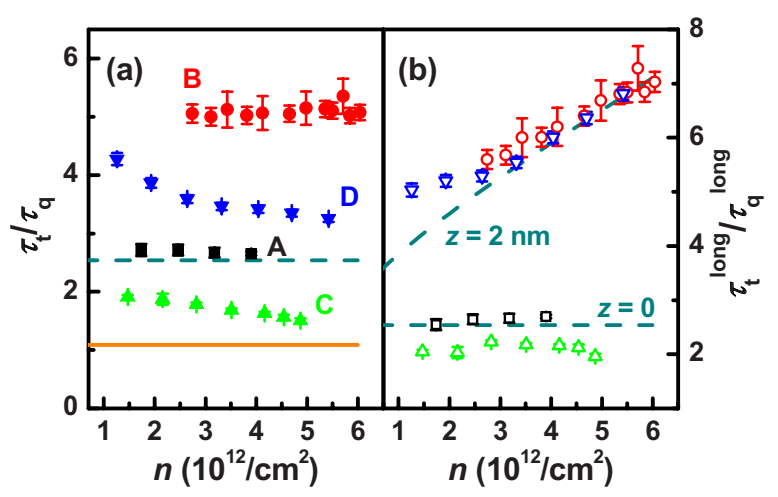

FIG. 3. (Color online) (a) $\tau_{t} / \tau_{q}$ vs $n$ in samples A (squares), B (circles), $\mathrm{C}$ (up triangles), and D (down triangles) along with calculations for $\mathrm{CI}$ at $z=0$ (dashed line) and $\delta$-scattering centers (solid

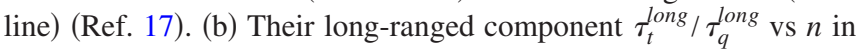
corresponding open symbols. Dashed lines are calculations for $z$ $=0$ (bottom) and $2 \mathrm{~nm}$ (top) (Ref. 17).

The $n$ dependence of $\tau_{q}$ in Fig. 2(c) agrees qualitatively with that of $\sigma\left(V_{\mathrm{g}}\right)$ in Fig. 1. This can be seen by separating the long and short-ranged components in $\tau_{t, q}$ using the following equations: ${ }^{17}$

$$
\begin{gathered}
\frac{1}{\tau_{t, q}}=\frac{1}{\tau_{t, q}^{\text {long }}}+\frac{1}{\tau_{t, q}^{\text {short }}}, \\
\tau_{t}^{\text {short }}=\frac{m^{*}}{n e^{2} \rho_{\text {short }}} ; \quad \frac{\tau_{t}^{\text {short }}}{\tau_{q}^{\text {short }}}=1.1 .
\end{gathered}
$$

Due to a small $\rho_{\text {short }}$, both $\tau_{t}$ and $\tau_{q}$ in sample A follow closely the $\sqrt{n}$ dependence expected for charged impurities residing in the graphene plane $(z=0) .{ }^{17}$ Large $\rho_{\text {short }}$ and higher $\mu_{\mathrm{FE}}$ in samples B-D (Table I) cause both scattering times to deviate from the $\sqrt{n}$ dependence. However, $\tau_{q}$ does not correlate with $\mu_{\mathrm{FE}}$ in a simple relation. Samples B and C exhibit similar $\mu_{\mathrm{FE}}$, but their $\tau_{q}$ differ by a factor of 2 . Samples A and B show comparable $\tau_{q}$ s despite the significant difference in $\mu_{\mathrm{FE}}$. Sample $\mathrm{C}$, with a moderate $\mu_{\mathrm{FE}}$ and the highest $\rho_{\text {short }}$, exhibits the highest $\tau_{q}$.

To understand the above observations, we calculate the ratio $\tau_{t} / \tau_{q}(n)$ and the long-ranged component $\tau_{t}^{\text {long }} / \tau_{q}^{\text {long }}(n)$ using Eq. (4) and plot the results in Figs. 3(a) and 3(b) (also Table I). Samples A and B show $n$-independent $\tau_{t} / \tau_{q}$ of 2.7 and 5.1, respectively. For the other two samples, $\tau_{t} / \tau_{q}$ decreases slightly with increasing $n$, varying from 1.9 to 1.5 in sample $\mathrm{C}$ and 4.3 to 3.3 in sample D. Clearly, the angular distribution $\mathrm{Q}(\theta)$ in Eq. (1) differs significantly in these samples.

By evaluating $\tau_{t}^{\text {long }} / \tau_{q}^{\text {long }}(n)$, we find that such variation may be naturally explained by varying the impurity-graphene distance $z$ within the charged-impurity model. Theoretical calculations of short and long-ranged ratios are given in solid and dashed lines, respectively, in Figs. 3(a) and 3(b). ${ }^{3,17}$ Ac-

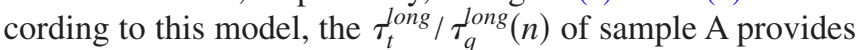
further evidence for the domination of CI located in the graphene plane $(z=0)$, where a constant 2.5 is expected (dashed line). ${ }^{3,17}$ The $\tau_{t} / \tau_{q}(n)$ in sample $\mathrm{C}$ falls between the dashed and solid lines, which is the result of a large shortranged component. Its $\tau_{t}^{\text {long }} / \tau_{q}^{\text {long }}(n)$ exhibits an approximate constant ratio of 2.1, also pointing to CI located in the graphene plane [Fig. 3(b)]. $\tau_{t}^{\text {long }} / \tau_{q}^{\text {long }}$ in samples B and D range from 5-7 and are best described by CI located $2 \mathrm{~nm}$ away from the graphene sheet [Fig. 3(b)]. In all six samples, we find $2<\tau_{t}^{\text {long }} / \tau_{q}^{\text {long }}<7$, corresponding to CI located within $2 \mathrm{~nm}$ of the graphene sheet.

The knowledge of the impurity-graphene distance $z$ is essential in correctly determining the CI density $n_{\text {imp }}$ in the vicinity of graphene. In the CI model, $n_{\text {imp }}$ is related to $\mu_{\mathrm{FE}}$ through $\mu_{\mathrm{FE}}=C / n_{\text {imp }}$, where $C=5 \times 10^{15} / \mathrm{V} \mathrm{s}$ is $n$-independent for $z=0 .{ }^{12}$ At a finite $z, C$ increases with increasing $n$ due to screening. Using the equations in Refs. 3 and 17, we numerically calculate $C(n, z)$ and estimate $n_{i m p}$ by fitting $\sigma(n)$ to Eq. (2). The results are listed in Table I while an exemplary fitting is given in Ref. 23. Clearly, $\mu_{\mathrm{FE}}\left(\right.$ or $\tau_{t}$ ) is affected by both $z$ and $n_{i m p}$. For example, the difference in $\mu_{\mathrm{FE}}$ between samples A and D mainly stems from $z$, instead of $n_{\text {imp }}$. In contrast, $\tau_{q}$ serves as a better measure of $n_{\text {imp }}$ due to its weaker dependence on $z$.

Next we briefly assess the effect of density inhomogeneity $\delta n$ on the measurement of $\tau_{q}$. Caused by CI, $\delta n$ measures approximately a few $10^{11} / \mathrm{cm}^{2}$ near the Dirac point ${ }^{16,26}$ and is expected to decrease with increasing $n$ due to electron screening. ${ }^{27} \delta n$ introduces phase smearing in $\rho_{\mathrm{xx}}(B)$, effectively reducing the $\mathrm{SdH}$ oscillation amplitude and suppressing the value of $\tau_{q}$ determined through the Dingle plot, as demonstrated in GaN 2DEGs. ${ }^{21}$ We have used the intercept of the Dingle plot at $1 / B=0$ as a criterion ${ }^{20}$ to obtain $\delta n$ and the corresponding corrections to $\tau_{q}$. Details are given in Ref. 23. We estimate $\delta n$ to be $\sim 7 \times 10^{10} / \mathrm{cm}^{2}$ in sample A, and $\sim 9 \times 10^{10} / \mathrm{cm}^{2}$ in sample B. These estimates are consistent with the highest filling factors observed in these samples $(\nu$ $=46$ for sample A and $\nu=74$ for sample B). Overall, $\delta n / n$ decreases rapidly with $n$, in agreement with theory; but the magnitude is only a few percent in the density range we studied, which is significantly smaller than the theoretical predictions..$^{23,27,28}$ The above correction leads to $\sim 20 \%$ increase of $\tau_{q}$ in sample A and $50 \%$ in sample B. The corrected $\tau_{q} \mathrm{~s}$ are given in Table I in parentheses. In samples $\mathrm{C}$ and $\mathrm{D}$, the corrections are smaller than the error bars of $\tau_{q}$ and there-

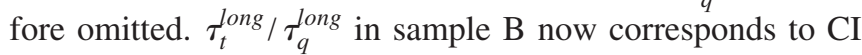
located at $z=1 \mathrm{~nm}$ instead of previously determined $z$ $=2 \mathrm{~nm}$, but the main picture does not change. ${ }^{23}$

Our study of $\tau_{q}$ and $\tau_{t} / \tau_{q}$ provides critical information in differentiating various scattering scenarios in graphene. ${ }^{4-7,17}$ A detailed comparison to theory is only made for the CI model at this point, but can be extended to other proposals as quantitative predictions become available. The diverse behavior our samples exhibit can all be understood very well within the CI model using three parameters: $n_{\text {imp }}, z$, and $\rho_{\text {short }}$. We speculate that uncontrolled spatial variation of $\mathrm{SiO}_{2}$ surface properties, as well as sample preparation conditions (e.g., humidity) may have been the primary reasons behind the observed differences among samples, although variations in preparation procedures cannot be ruled out. ${ }^{23}$

Our results indicate that the dominant CI resides within 2 $\mathrm{nm}$ of and sometimes in the immediate vicinity of the graphene sheet. Primary candidates of this nature are charges 
carried by adsorbates on top of graphene and/or at the graphene $/ \mathrm{SiO}_{2}$ interface. The role of adsorbates is especially highlighted in the current annealing treatment of suspended graphene. ${ }^{10,11}$ In the literature, various approaches, including resist-free processing, ${ }^{16,29,30}$ UHV baking, ${ }^{13,16}$ and current annealing, ${ }^{31}$ have been used to remove contaminants on top of graphene without significant improvement to mobility. These observations collectively suggest that adsorbates on top of graphene cannot be the major culprit in limiting mobility at the current level. Instead, we speculate that charges (e.g., $\mathrm{Na}^{+}$) and molecular groups (e.g., $\mathrm{OH}$ ) adsorbed/trapped on the $\mathrm{SiO}_{2}$ surface prior to the exfoliation of graphene are the major source of scattering. The $z=0$ found in samples A and $\mathrm{C}$ lends strong support to this hypothesis. Moreover, the small $z$ observed in other samples can be accounted for by the existence of a spacer layer between graphene and $\mathrm{SiO}_{2}$ (e.g., $\mathrm{H}_{2} \mathrm{O}$ ). The evidence of such a layer is widely observed in AFM height measurements of graphene. In this scenario, the concentration of adsorbed charges $\left(n_{\text {imp }}\right)$, together with the thickness of the spacer layer $(z)$ can account for the wide span of mobility seen in our samples. It may also explain why graphene on a variety of substrates displays a similar range of mobility ${ }^{15}$ since the bulk properties of these substrates are less relevant here.
Finally we note that the above determined $z$ can be expanded to represent an average impurity-graphene distance. Using this concept, we consider the contribution of uniformly distributed charges within the bulk of the $\mathrm{SiO}_{2}$ substrate. Our simulations show that oxide charges in commercially available $\mathrm{SiO}_{2}$ are unlikely to be a major source of scattering at the current mobility level. ${ }^{23}$

In conclusion, we have systematically studied the quantum and transport scattering times in graphene. Our data will prove useful in critical examinations of existing scattering scenarios. Within the CI model, the ratio of $\tau_{t} / \tau_{q}$ indicates that charged impurities residing within $2 \mathrm{~nm}$ of the graphene sheet are the main sources of scattering in graphene. Such information provides important guidance to the effort of improving carrier mobility in graphene.

We are grateful for helpful discussions with S. Adam and L. Song, and technical assistance from S.-H. Cheng and S. Syed. We thank P. Eklund for providing access to his Raman spectrometer. Work at Penn State is supported by NSF Grants No. NIRT ECS-0609243, No. CAREER DMR0748604, and No. MRSEC DMR-0820404. The authors acknowledge use of facilities at the PSU site of NSF NNIN.
${ }^{1}$ T. Ando, J. Phys. Soc. Jpn. 75, 074716 (2006).

${ }^{2}$ K. Nomura and A. H. MacDonald, Phys. Rev. Lett. 98, 076602 (2007).

${ }^{3}$ S. Adam, E. H. Hwang, V. M. Galitski, and S. Das Sarma, Proc. Natl. Acad. Sci. U.S.A. 104, 18392 (2007).

${ }^{4}$ T. O. Wehling, A. V. Balatsky, M. I. Katsnelson, A. I. Lichtenstein, K. Scharnberg, and R. Wiesendanger, Phys. Rev. B 75, 125425 (2007).

${ }^{5}$ T. Stauber, N. M. R. Peres, and F. Guinea, Phys. Rev. B 76, 205423 (2007).

${ }^{6}$ M. I. Katsnelson and A. K. Geim, Philos. Trans. R. Soc. London, Ser. A 366, 195 (2008).

${ }^{7}$ M. I. Katsnelson, F. Guinea, and A. K. Geim, Phys. Rev. B 79, 195426 (2009).

${ }^{8}$ Y. W. Tan, Y. Zhang, K. Bolotin, Y. Zhao, S. Adam, E. H. Hwang, S. Das Sarma, H. L. Stormer, and P. Kim, Phys. Rev. Lett. 99, 246803 (2007).

${ }^{9}$ F. Schedin, A. K. Geim, S. V. Morozov, E. W. Hill, P. Blake, M. I. Katsnelson, and K. S. Novoselov, Nature Mater. 6, 652 (2007).

${ }^{10}$ K. I. Bolotin, K. J. Sikes, Z. Jiang, M. Klima, G. Fudenberg, J. Hone, P. Kim, and H. L. Stormer, Solid State Commun. 146, 351 (2008)

${ }^{11}$ X. Du, I. Skachko, A. Barker, and E. Andrei, Nat. Nanotechnol. 3, 491 (2008).

${ }^{12}$ J. H. Chen, C. Jang, S. Adam, M. S. Fuhrer, E. D. Williams, and M. Ishigami, Nat. Phys. 4, 377 (2008).

${ }^{13}$ C. Jang, S. Adam, J. H. Chen, E. D. Williams, S. Das Sarma, and M. S. Fuhrer, Phys. Rev. Lett. 101, 146805 (2008).

${ }^{14}$ X. Hong, A. Posadas, K. Zou, C. H. Ahn, and J. Zhu, Phys. Rev. Lett. 102, 136808 (2009).

${ }^{15}$ L. A. Ponomarenko, R. Yang, T. M. Mohiuddin, M. I. Katsnelson, K. S. Novoselov, S. V. Morozov, A. A. Zhukov, F. Schedin,
E. W. Hill, and A. K. Geim, Phys. Rev. Lett. 102, 206603 (2009).

${ }^{16}$ Y. Zhang, V. W. Brar, C. Girit, A. Zettl, and M. F. Crommie, Nat. Phys. 5, 722 (2009).

${ }^{17}$ E. H. Hwang and S. Das Sarma, Phys. Rev. B 77, 195412 (2008).

${ }^{18}$ S. Das Sarma and F. Stern, Phys. Rev. B 32, 8442 (1985).

${ }^{19}$ J. P. Harrang, R. J. Higgins, R. K. Goodall, P. R. Jay, M. Laviron, and P. Delescluse, Phys. Rev. B 32, 8126 (1985).

${ }^{20}$ P. T. Coleridge, Phys. Rev. B 44, 3793 (1991).

${ }^{21}$ S. Syed, M. J. Manfra, Y. J. Wang, R. J. Molnar, and H. L. Stormer, Appl. Phys. Lett. 84, 1507 (2004).

${ }^{22}$ Z. Jiang, E. A. Henriksen, L. C. Tung, Y. J. Wang, M. E. Schwartz, M. Y. Han, P. Kim, and H. L. Stormer, Phys. Rev. Lett. 98, 197403 (2007).

${ }^{23}$ See EPAPS Document No. E-PRBMDO-80-R32948 for supporting information. For more information on EPAPS, see http:// www.aip.org/pubservs/epaps.html

${ }^{24}$ M. Trushin and J. Schliemann, Phys. Rev. Lett. 99, 216602 (2007).

${ }^{25}$ B. Huard, N. Stander, J. A. Sulpizio, and D. Goldhaber-Gordon, Phys. Rev. B 78, 121402(R) (2008).

${ }^{26}$ J. Martin, N. Akerman, G. Ulbricht, T. Lohmann, J. H. Smet, K. von Klitzing, and A. Yacoby, Nat. Phys. 4, 144 (2008).

${ }^{27}$ E. Rossi and S. Das Sarma, Phys. Rev. Lett. 101, 166803 (2008).

${ }^{28}$ M. Polini, A. Tomadin, R. Asgari, and A. H. MacDonald, Phys. Rev. B 78, 115426 (2008).

${ }^{29}$ N. Staley, H. Wang, C. Puls, J. Forster, T. N. Jackson, K. McCarthy, B. Clouser, and Y. Liu, Appl. Phys. Lett. 90, 143518 (2007).

${ }^{30}$ C. O. Girit and A. Zettl, Appl. Phys. Lett. 91, 193512 (2007).

${ }^{31}$ J. Moser, A. Barreiro, and A. Bachtold, Appl. Phys. Lett. 91, 163513 (2007). 